\title{
Developments in the meiotic analysis of hybrids. II. Amended models for tetraploids
}

\author{
C. G. D. CHAPMAN \& G. KIMBER* \\ PBI CAMBRIDGE, Maris Lane, Trumpington, Cambridge CB2 2LQ, U.K. *Department of Agronomy, Curtis Hall, \\ University of Missouri-Columbia, M.O. 65211, U.S.A.
}

\begin{abstract}
Amended models of meiotic behaviour in tetraploid species' hybrids have been constructed which better reflect the theory and assumptions about chromosome pairing necessary to conduct such analyses. In particular they correct problems concerning the distribution of chiasmata among and within chromosome configurations inherent in the earlier models of Kimber \& Alonso (1981). In general these analyses give similar interpretations to the earlier models but in some cases call the previous conclusions into question.
\end{abstract}

Keywords: meiotic analysis, pairing models, tetraploids.

\section{Introduction}

The analysis of chromosome pairing in species hybrids is a fundamental part of the studies in the classification and evolution of polyploid taxa in plants. The early work, particularly that of Kihara (see Lilienfeld, 1951), made a somewhat qualitative assessment of the pairing patterns observed at metaphase I of meiosis. To the extent that bivalents were seen in approximate multiples of the basic chromosome number. It was concluded, therefore, that the parental species had that number of genomes in common.

In recent years more objective, mathematical approaches to quantify the patterns of chromosome pairing have been developed. One by Driscoll et al. (1979) distinguishes patterns of association from levels of pairing. These concepts were extended by Alonso \& Kimber (1981), Kimber \& Alonso (1981) and Espinasse \& Kimber (1981) to assess the relative similarity or affinity amongst the genomes in triploid, tetraploid and pentaploid hybrids, respectively. This has allowed fresh insights into chromosome pairing often confirming, but sometimes upsetting, conclusions based on subjective assessments. For example, Kimber et al. (1983) were able to show that the $\mathrm{M}^{\mathrm{u}}$ genome of Triticum uniaristatum bore no particular resemblance to the $\mathrm{M}$ genome of $T$. comosum. It was therefore assigned the symbol Un, which has subsequently been reduced to the single letter $\mathrm{N}$ (Kimber \& Tsunewaki, 1988).

For tetraploid hybrids Kimber \& Alonso (1981) proposed three different models of pairing. These are designated $2: 2,2: 1: 1$, and $3: 1$, with two pairs, one pair, and a triplet of equally closely related (not necessarily homologous) genomes respectively. The remaining relationships are considered equal and more distant (Fig. 1). The relative affinity of the more closely related genomes is designated $x$, and that of the less related genomes $y$, where $x>y$ and $x+y=1$. Equations were developed for the expected frequencies of each type of meiotic figure taking into account not only $x$ but also the mean arm-pairing frequency, $c$, which can be determined by direct observation. A computer program optimizes $x$ for each model by varying $x$ and searching for the best fit as determined by the minimum sums of squares of difference (SSD) between observed and calculated figure numbers. In choosing among the three models after optimization, that which gave the smallest SSD is generally preferred. There is a fourth possible model, $4: 0$, which corresponds to the genomic formula $\mathrm{AAAA}$, when all the genomes are identical, or $\mathrm{ABCD}$, when they are equally unrelated. This may be regarded as a special example of the other three models as they converge on $x=y=0.5$.

The tetraploid formulae developed by Driscoll $e t a l$. (1979), and Kimber \& Alonso (1981), have been criticized by Jackson \& Casey (1982) and Crane \& Sleper (1989) concerning the placement of the second pair of bound arms in configurations. The problem is best understood in the context of an autotetraploid where the affinities amongst all four chromosomes are equal. The former group of authors argue that, following the establishment of one set of bound arms, there remain seven other possible ways of arm pairing (one on the same side of the centromere and six involving the other 


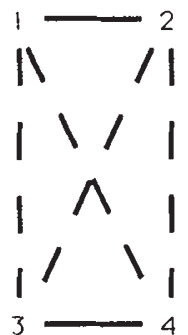

$2: 2$

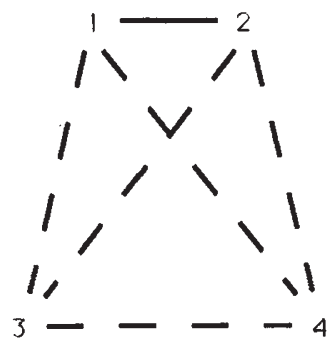

2:1:1

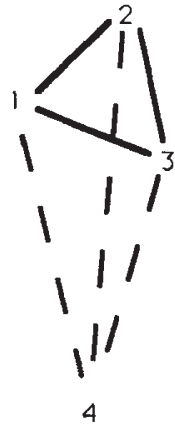

$3: 1$
Fig. 1 The three basic models of pairing in tetraploid hybrids. (_-) More closely related genomes, relative affinity $=x .(--)$ Less closely related genomes, relative affinity $=y$. set of arms) and that each is equally possible. The latter group contend that whilst a further one pair of bound arms might form on the same side, the maximum possible on the far side is two and that the probabilities of placement of the second binding are in the same proportions. The net effect is to change the ratio of 'near-side second binding' to 'far-side second binding' from $1: 6$ to $1: 2$ and change the frequency of the resulting configurations accordingly. Crane \& Sleper (1989) have developed a formal proof to substantiate their opinion, with which we concur, as the former view implies interference across the centromere which the theory behind the models expressly excludes.

An additional problem has been identified in connection with the distribution of paired arms. The models of Kimber \& Alonso (1981) partition the number of paired arms a priori amongst meiotic configurations (set of four homoeologous chromosomes) according to the expansion of the binomial $[c+(1-c)]^{4}$, where $c$ is the mean arm pairing frequency, irrespective of the model and value of $x$. Thus, if $c=0.5$ then configurations are expected to have 0,1 , 2, 3 and 4 paired arms in the ratio 0.0625 : $0.2500: 0.3750: 0.2500: 0.0625$. In the case of the ideal $2: 1: 1$ hybrid, however, where the more closely related genomes are fully homologous and completely paired and the others have no homology and remain unpaired, each homoeologous group will form one ring bivalent and two univalents. While $c=0.5$, every configuration will have two paired arms only, and the complex ratios of meiotic figures, predicted by the 2:1:1 model of Alonso \& Kimber (1981), will not be realized (see Table 1). A similar difficulty arises with the $3: 1$ model. Furthermore, their solution to an ideal amphidiploid, where all the figures are ring bivalents, is ambiguous because a perfect fit is also given by the 2:1:1 model (Table 1). In some cases this problem may adversely influence the optimized value of $x$ and/or lead to the wrong choice among models.

In addition Chapman \& Kimber (1992a) have proposed that in the course of optimization the numbers of meiotic figures should be weighted (multiplied) by the numbers of chromosomes in each. The value of $x$ then sought is that which minimizes this weighted sums of squares of difference (WSSD) between observed and calculated figure frequencies. This removes a bias in the estimate of $x$ towards solutions favoured by the smaller figures. In light of these considerations the models for chromosome pairing in tetraploid hybrids have been re-examined and amended. These are reported below. The terms pairing, meiotic figure, meiotic configuration, arm configuration, dataset, meiotic analysis and $c$ are used as discussed by Chapman \& Kimber (1990a).

\section{Theory}

As with the models of Kimber \& Alonso (1981) and Crane \& Sleper (1989), a number of assumptions and simplifications are made about the nature of chromosome pairing. These were discussed by Chapman \& Kimber (1992a) and are summarized below.

The first assumption is that the pairing of the two arms of a chromosome may be considered independent and hence the configurations that result are the product of their individual behaviour. Secondly, the pairing of one arm with another precludes any involvement of a third. It is also assumed that the role of any translocations present in generating multivalents is negligable. Finally, the affinity relationships and tendency to pair of any four homoeologous arms are the same as those for the genomes as a whole.

In conformity with the models of Kimber \& Alonso (1981), the relative affinity of the more closely related genomes is defined as $x$, and that of the less closely related genomes is defined as $y$, where $x>y$ and $x+y=1$. In tetraploids any homoeologous group may form up to four arm pairs. From this it follows that the mean arm-pairing frequency, $c$, may be calculated from an observed dataset as (rod bivalents $+2 \times$ (ring bivalents + trivalents $)+3 \times$ open quadrivalents $+4 \times$ closed quadrivalents $) /(4 \times$ basic number $)$. 
Table 1 Comparison of the present models with those of Kimber \& Alonso (1981) for idealized datasets for tetraploid hybrids with a basic number of 7

\begin{tabular}{|c|c|c|c|c|c|c|c|c|c|c|}
\hline \multirow{2}{*}{$\begin{array}{l}\text { Representative } \\
\text { genomes }\end{array}$} & Dataset* & $\mathrm{I} \dagger$ & \multirow{2}{*}{$\frac{\lfloor\text { II }\rfloor}{0.00}$} & \multirow{2}{*}{ (II) } & \multirow{2}{*}{$\begin{array}{l}\text { III } \\
0.00\end{array}$} & \multirow{2}{*}{$\frac{\lfloor\mathrm{IV}}{0.00}$} & \multirow{2}{*}{ (IV) } & \multicolumn{2}{|c|}{$\begin{array}{l}\text { Pairing/ } \\
\text { merit function }\end{array}$} & \multirow[t]{2}{*}{$x$} \\
\hline & Ideal $4: 0$ & 0.00 & & & & & & $c \ddagger$ & 1.000 & \\
\hline \multirow[t]{2}{*}{$\mathrm{ABCD}$} & K/A $\quad 4: 0$ & 0.00 & 0.00 & 4.67 & 0.00 & 0.00 & 4.67 & SSD & 0.000 & 0.500 \\
\hline & $\mathrm{C} / \mathrm{K} \quad 4: 0$ & 0.00 & 0.00 & 4.67 & 0.00 & 0.00 & 4.67 & WSSD & 0.000 & 0.500 \\
\hline \multirow[t]{5}{*}{$\mathrm{AABB}$} & Ideal $2: 2$ & 0.00 & 0.00 & 14.00 & 0.00 & 0.00 & 0.00 & $c$ & 1.000 & \\
\hline & K/A $2: 2$ & 0.00 & 0.00 & 14.00 & 0.00 & 0.00 & 0.00 & SSD & 0.000 & 1.000 \\
\hline & K/A $2: 1: 1$ & 0.00 & 0.00 & 14.00 & 0.00 & 0.00 & 0.00 & SSD & 0.000 & 1.000 \\
\hline & $\mathrm{C} / \mathrm{K} \quad 2: 2$ & 0.00 & 0.00 & 14.00 & 0.00 & 0.00 & 0.00 & WSSD & 0.000 & 1.000 \\
\hline & $\mathrm{C} / \mathrm{K} \quad 2: 1: 1$ & 0.00 & 0.00 & 4.67 & 0.00 & 0.00 & 4.67 & WSSD & 696.887 & 0.500 \\
\hline \multirow[t]{3}{*}{$\mathrm{AABC}$} & Ideal $2: 1: 1$ & 14.00 & 0.00 & 7.00 & 0.00 & 0.00 & 0.00 & $c$ & 0.500 & \\
\hline & K/A $\quad 2: 1: 1$ & 10.48 & 3.51 & 5.23 & 0.01 & 0.01 & 0.00 & SSD & 27.820 & 1.000 \\
\hline & $\mathrm{C} / \mathrm{K} \quad 2: 1: 1$ & 14.00 & 0.00 & 7.00 & 0.00 & 0.00 & 0.00 & WSSD & 0.000 & 1.000 \\
\hline \multirow[t]{3}{*}{$\mathrm{AAAB}$} & Ideal $3: 1$ & 9.33 & 0.00 & 2.33 & 4.67 & 0.00 & 0.00 & $c$ & 0.500 & \\
\hline & K/A $\quad 3: 1$ & 8.75 & 2.34 & 1.75 & 1.75 & 1.17 & 0.29 & SSD & 16.114 & 1.000 \\
\hline & $\mathrm{C} / \mathrm{K} \quad 3: 1$ & 9.33 & 0.00 & 2.33 & 4.67 & 0.00 & 0.00 & WSSD & 0.000 & 1.000 \\
\hline
\end{tabular}

${ }^{*} \mathrm{~K} / \mathrm{A}=$ analysis of the ideal dataset by the models of Kimber \& Alonso (1981); $\mathrm{C} / \mathrm{K}=$ analysis of the ideal dataset by present models.

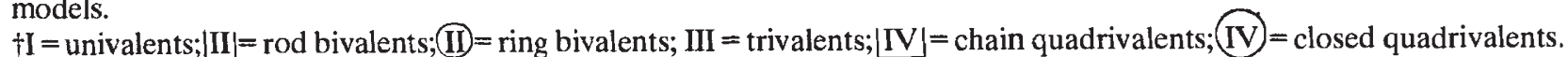
$\ddagger c=$ mean arm pairing frequency; $S S D=$ sums of squares of difference; WSSD $=$ weighted sums of squares of difference.

The structure of the 2:2, 2:1:1 and $3: 1$ models for a single set of four homoeologous chromosome arms is laid out in Table 2. The four genomes bear the same relationship to each other as illustrated in Fig. 1. With the amount of pairing expected in any set of arms being $2 c$ (half that for the whole chromosomes), and the relative affinities being reflected in the ratio of pairing amongst the six possible pairwise combinations, the frequency of pairing between any two arms $=$ $(2 c \times$ their relative affinity $) /(\Sigma$ relative affinities among all arms) (Table 2, ii).

There are three mutally exclusive patterns of pairing possible among the four arms. The first is described as (1-2,3-4), where only arms 1 and 2 , and/or 3 and 4 may pair; the others are correspondingly $(1-3,2-4)$ and $(1-4,2-3)$. Viewing the pairing of arms as being a sequential process, it follows that the ratio of these patterns will be the sums of the relative affinities of the two possible pairs of arms, since the first pairing will commit the set of arms to one of the three patterns (Table 2, ii).

Within any one of these patterns the proportion of any two arms pairing $=($ frequency of their pairing $) /($ frequency of the pattern), and the proportion of those two arms remaining unpaired $=(1-$ frequency of their pairing)/(frequency of the pattern) (Table 2, iii and iv). From this the proportion of each pattern with two, one (either pair) or no paired arms can be determined; and hence the frequency of the 10 possible 'arm configurations' for a homoeologous set of four chromosome arms for given values of $c$ and $x$ (Table $2, \mathrm{v}$ ).

The nine meiotic configurations that a single homoeologous group of four chromosomes can form ranges from four univalents to a ring quadrivalent or two ring bivalents depending upon the amount of pairing. Their frequencies may be determined by establishing a $10 \times 10$ array and siting the arm configurations and their frequencies along the margins. For each cell within the array the resulting meiotic configuration can be established from the marginal arm configurations, and its frequency as the product of the marginal frequencies. Summing for each configuration type over the whole array gives its frequency, and hence the frequencies of each meiotic figure (univalents through ring quadrivalents).

The optimization process is performed as described for the triploid model (Chapman \& Kimber, 1992a) by first calculating $c$ from the observed frequencies of figures, and then searching the permissible range of $x$ $(0.5-1.0)$ for that value which gives the minimum WSSD between the observed and calculated numbers of meiotic figures. Unlike the triploid model, analytical solutions are not possible.

A computer program has been developed to carry out the calculations described above. It is written in Turbo Pascal and runs on the Apple Macintosh. 


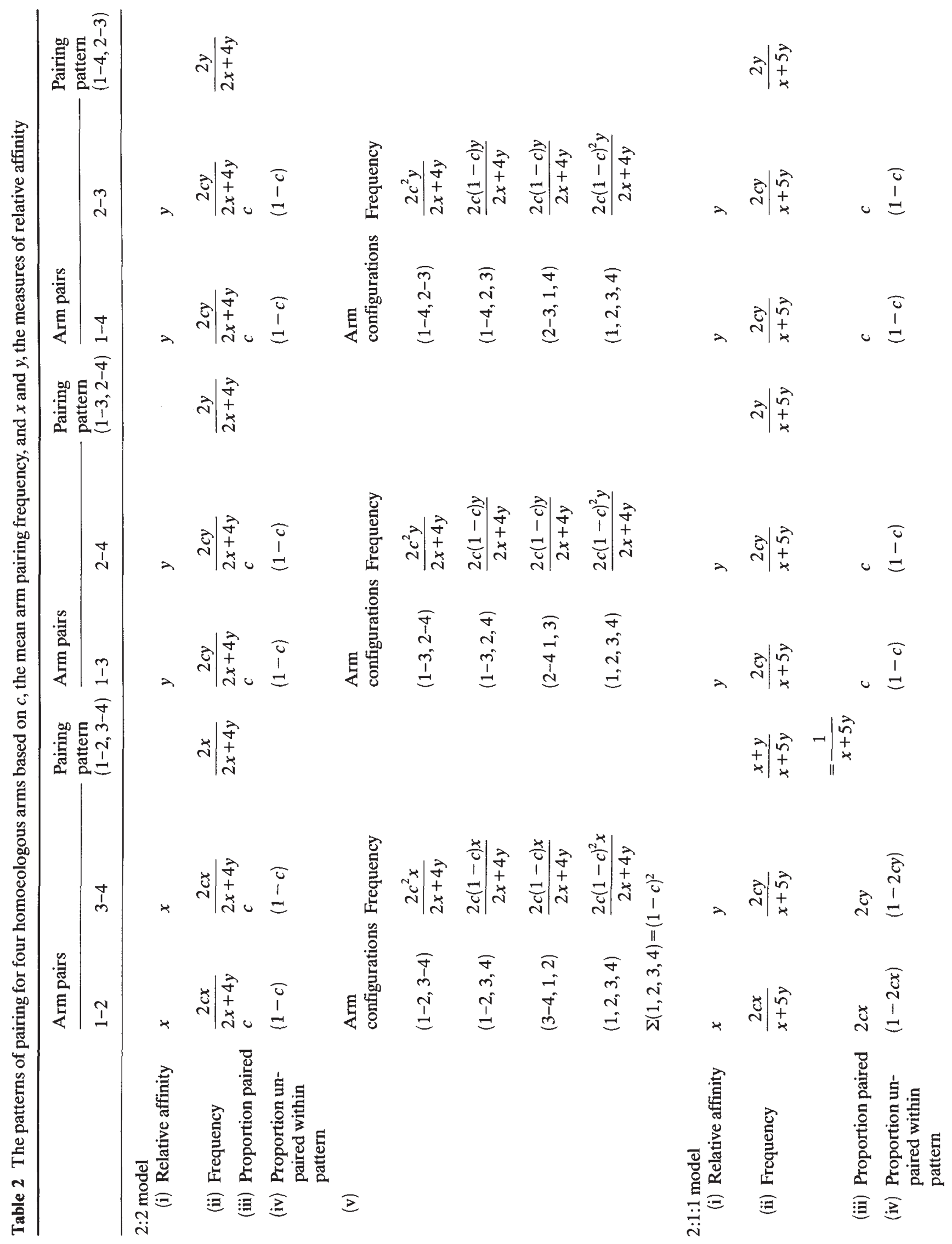




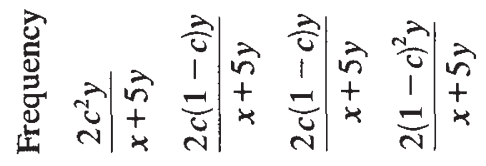

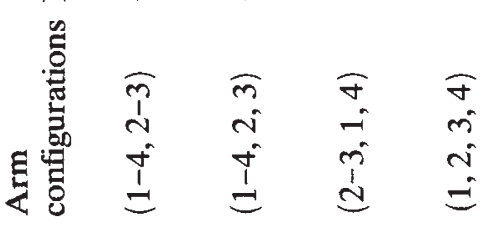

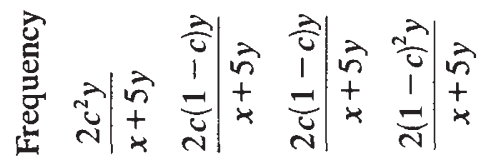

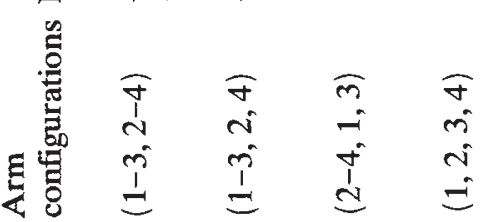

$+\underset{*}{+} \mid \begin{array}{cc}m & \\ + & -1 m \\ \infty & \| 1\end{array}$

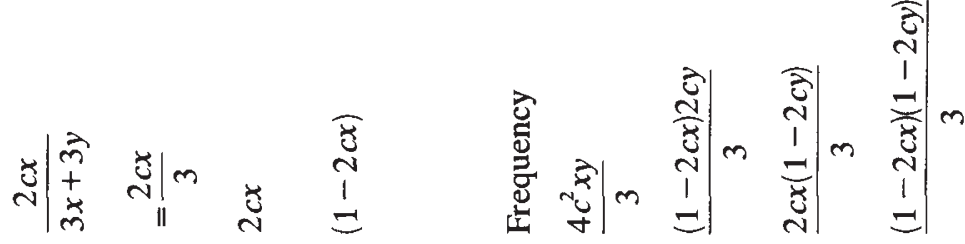

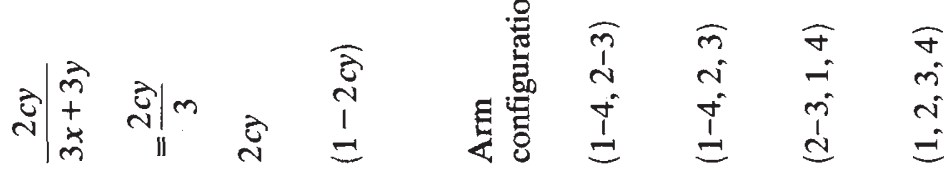

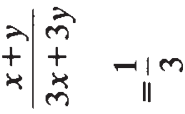

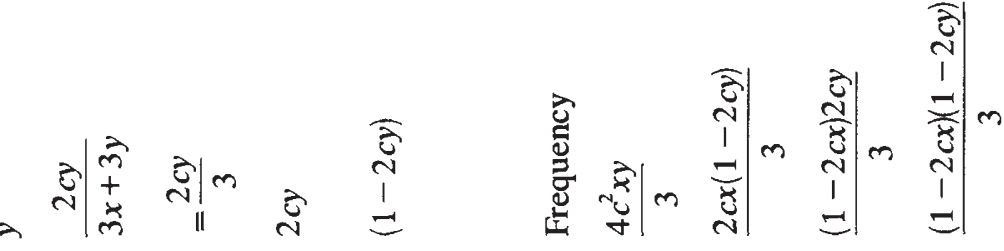

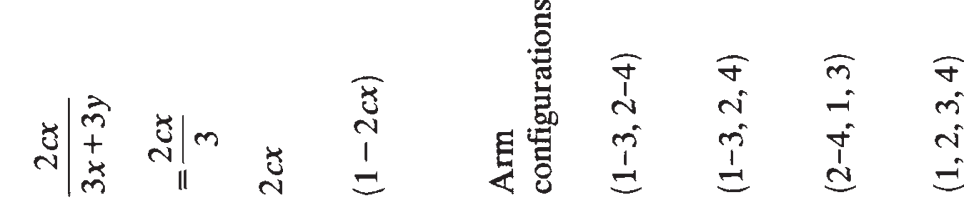

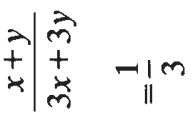

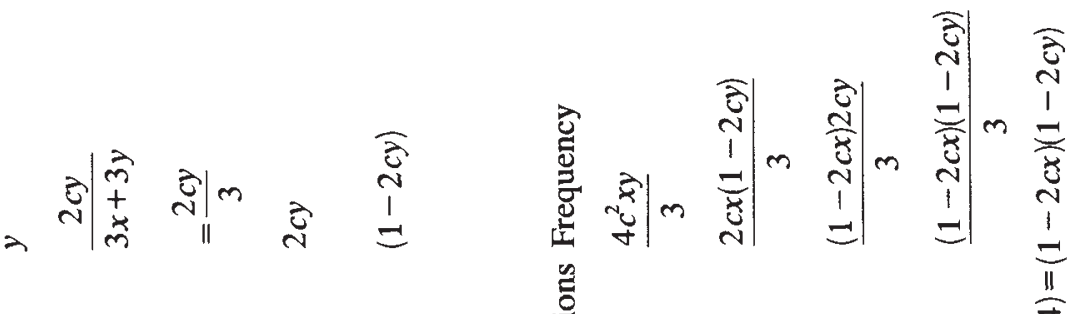

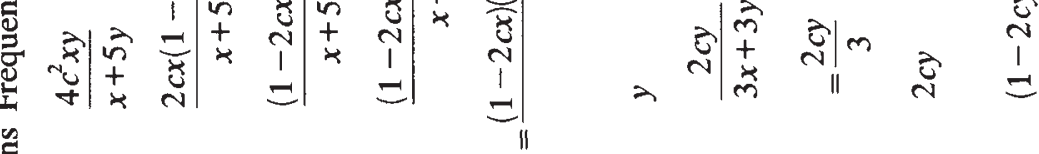

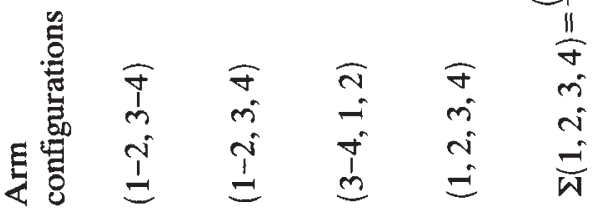

亏ृ

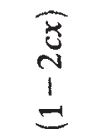

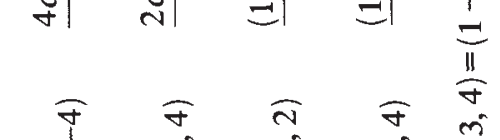




\section{Results and discussion}

When applied to theoretical datasets for perfect $4: 0$, $2: 2,2: 1: 1$ and $3: 1$ hybrids, the respective models optimize to exact fits with WSSDs of zero (see Table 1). For the 2:1:1 and 3:1 models, and unlike those of Kimber \& Alonso (1981), when $x=1, c$ cannot exceed 0.5 because the expression $1-2 c x$, used to calculate proportions of unpaired arms (Table 2), then becomes negative. It follows that for the $2: 1: 1$ and $3: 1$ models $x \leq 1 /(2 c)$. Thus if $c=1.0$ the only acceptable model is $2: 2$, except for the special case of the $4: 0$ hybrid, where the solution is $x=y=0.5$ for all models.

Figure 2 shows how the number of figures vary with $x$ when the basic number $=7$ and $c=0.607$ for all three models. These values have been chosen for direct comparison with Figs 1d, 2d and 3d of Kimber \& Alonso (1981) where the basic number also equals 7, and 17 out of a maximum 28 arm pairs have formed. It is apparent for the 2:2 model that as $x$ increases the frequency of the bivalents of both types increases, and that of the larger multivalents declines. The frequency of univalents is unaffected. The plots for the $2: 1: 1$ and $3: 1$ models terminate at $x=1 /(2 \times 0.607)=0.824$, beyond which the expression $(1-2 c x)$ becomes negative. In the $2: 1: 1$ model the frequencies of univalents, ring bivalents and trivalents increases with $x$, whereas those of rod bivalents and quadrivalents decline. The same general trends are also true of the 3:1 model, although the slopes for the ring bivalents and trivalents differ markedly.

In comparison with the models of Kimber \& Alonso (1981) the slopes are generally smoother, and for the 2:1:1 and 3:1 models do not carry through to $x=1$. Whereas the numbers of trivalents decline slightly in the former 2:1:1 model, here they rise slightly. The contrast is greatest in the 3:1 model, where Kimber \& Alonso (1981) suggested that quadrivalent frequencies were independent of $x$, whilst the present model shows a gentle decline for ring quadrivalents and a slight peak for rod quadrivalents around $x=0.7$. Furthermore, the rising slope for trivalents and the declining slope for rod bivalents are steeper. In one important respect all models are similar, in that the slopes for all figures flatten around $x=0.5$. This means that a small change in the observed numbers of figures in this region can result in a large change in the estimated value of $x$, and may well affect the choice of best-fit model.

Table 3 compares some analyses carried out under the models of Kimber \& Alonso (1981) with the present ones, presenting the better fits only. It should be noted that the SSD calculated by Kimber \& Alonso (1981) is not comparable to the WSSD calculated here, due to the weighting applied to the numbers of figures.
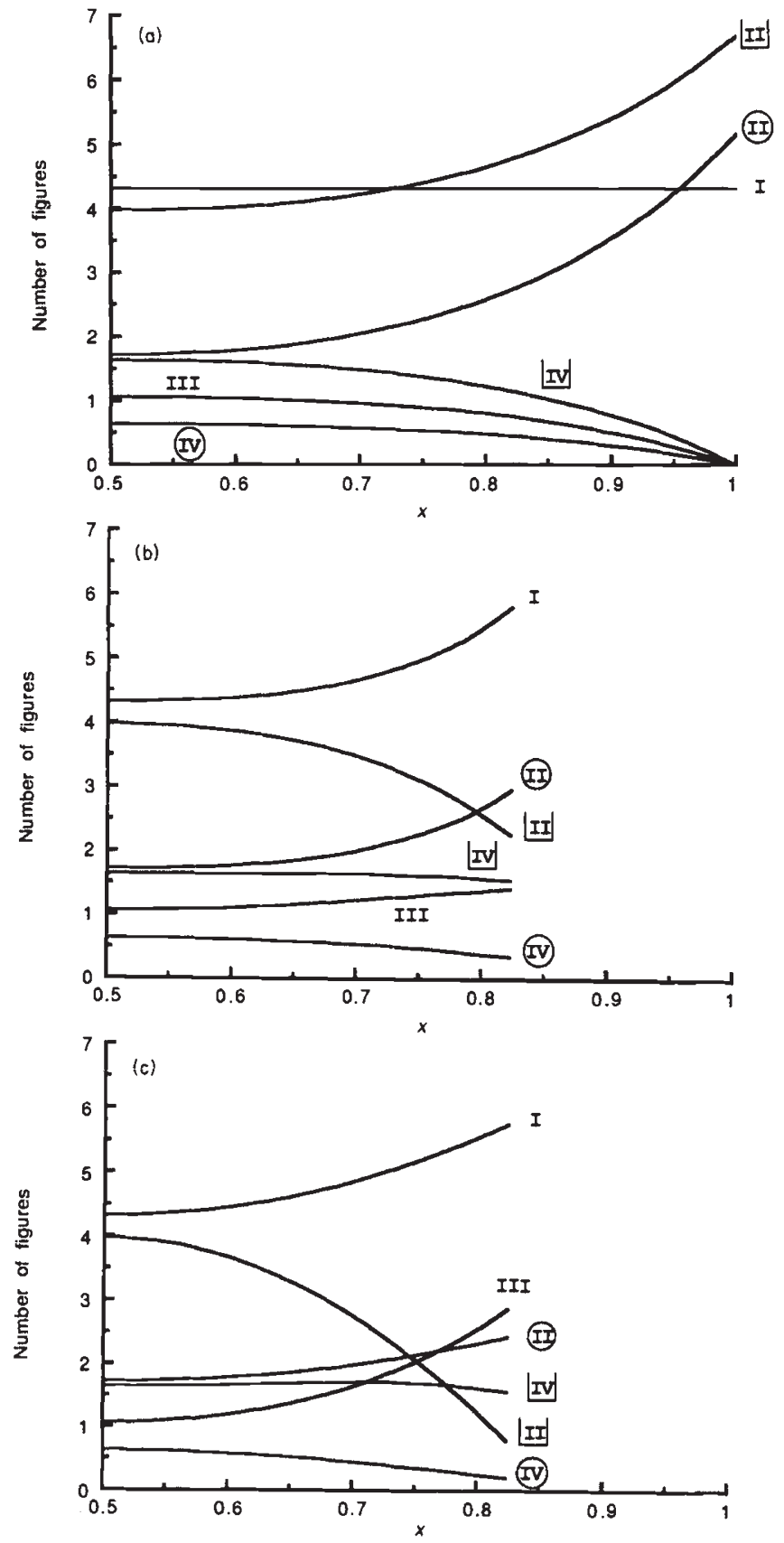

Fig. 2 The variation of expected numbers of meiotic figures with $x$ for the (a) 2:2, (b) 2:1:1 and (c) 3:1 models, when $c=0.607$.

Any comparison between the analyses must be done subjectively.

The first four entries are known examples of the 4:0, $2: 2,2: 1: 1$ and $3: 1$ models. Both analytical approaches agree on the choice of model and would give the same general interpretation of the observations. Inspection, however, suggests the present analyses give a better fit to the observed data, especially for the $2: 1: 1$ and $3: 1$ models. Furthermore, the values of $x$ estimated differ 
Table 3 Comparison of the present analyses with those of Kimber \& Alonso (1981)

\begin{tabular}{|c|c|c|c|c|c|c|c|c|c|c|}
\hline Hybrid and genomes & Dataset & I & III & (II) & III & IV & (IV) & $c$ & SSD/WSSD & $x$ \\
\hline Autotetraploid & Obs.* & 0.06 & 0.08 & 4.44 & 0.06 & 0.06 & 4.68 & 0.992 & & \\
\hline Hordeum bulbosum & K/A 4:0 & 0.00 & 0.07 & 4.60 & 0.00 & 0.13 & 4.53 & & 0.070 & 0.500 \\
\hline BBBB & $\mathrm{C} / \mathrm{K} 4: 0$ & 0.00 & 0.01 & 4.66 & 0.00 & 0.01 & 4.65 & & 0.297 & 0.500 \\
\hline Triticum boeoticum & Obs. & 0.40 & 3.15 & 9.00 & 0.30 & 0.35 & 0.25 & 0.850 & & \\
\hline$\times T$. tauschii amph. & K/A 2:2 & 0.68 & 2.84 & 9.20 & 0.16 & 0.51 & 0.17 & & 0.267 & 0.899 \\
\hline AADD & $\mathrm{C} / \mathrm{K} 2: 2$ & 0.63 & 3.25 & 9.05 & 0.05 & 0.27 & 0.38 & & 1.064 & 0.972 \\
\hline T. aestivum $\times$ & Obs. & 14.46 & 2.14 & 4.40 & 0.00 & 0.00 & 0.00 & 0.390 & & \\
\hline T. tauschii & K/A 2:1:1 & 13.55 & 3.49 & 3.71 & 0.00 & 0.00 & 0.00 & & 3.155 & 0.999 \\
\hline ABDD & $\mathrm{C} / \mathrm{K} 2: 1: 1$ & 14.67 & 2.39 & 4.27 & 0.00 & 0.00 & 0.00 & & 0.360 & 1.000 \\
\hline T. triunciale $\times 4 x$ & Obs. & 9.40 & 2.35 & 1.45 & 2.65 & 0.70 & 0.00 & 0.452 & & \\
\hline T. umbellulatum & K/A 3:1 & 10.01 & 2.71 & 1.47 & 1.69 & 0.94 & 0.19 & & 1.510 & 0.977 \\
\hline UUUC & C/K 3:1 & 9.61 & 2.58 & 1.55 & 2.63 & 0.53 & 0.03 & & 0.737 & 0.896 \\
\hline T. crassum $\times$ & Obs. & 13.94 & 4.06 & 1.46 & 0.81 & 0.09 & 0.03 & 0.321 & & \\
\hline T. umbellulatum & K/A 2:1:1 & 14.03 & 3.98 & 1.35 & 0.77 & 0.23 & 0.02 & & 0.048 & 0.909 \\
\hline DDMU & $\mathrm{C} / \mathrm{K} 2: 1: 1$ & 14.03 & 4.06 & 1.42 & 0.70 & 0.21 & 0.01 & & 0.361 & 0.917 \\
\hline T. juvenale $\times$ & Obs. & 13.14 & 4.17 & 0.42 & 1.40 & 0.35 & 0.03 & 0.320 & & \\
\hline T. longissimum & $\mathrm{K} / \mathrm{A} 4: 0$ & 13.30 & 4.16 & 0.54 & 1.14 & 0.42 & 0.05 & & 0.133 & 0.500 \\
\hline DMUS $^{1}$ & C/K 3:1 & 13.32 & 4.14 & 0.68 & 1.27 & 0.29 & 0.02 & & 0.518 & 0.822 \\
\hline T. aestivum $\times$ & Obs. & 12.35 & 3.35 & 1.65 & 1.55 & 0.25 & 0.00 & 0.375 & & \\
\hline Secale cereale & K/A 3:1 & 12.28 & 3.10 & 1.09 & 1.50 & 0.62 & 0.09 & & 0.521 & 0.959 \\
\hline ABDR & C/K 3:1 & 11.72 & 3.55 & 1.05 & 1.89 & 0.34 & 0.01 & & 3.180 & 0.887 \\
\hline T. timopheevii $\times$ & Obs. & 7.60 & 4.20 & 3.60 & 1.20 & 0.30 & 0.00 & 0.525 & & \\
\hline T. turgidum & K/A 2:2 & 6.65 & 4.94 & 3.63 & 1.00 & 0.71 & 0.10 & & 2.608 & 0.812 \\
\hline \multirow[t]{3}{*}{$\mathrm{AABG}$} & K/A 2:1:1 & 7.96 & 3.79 & 3.32 & 0.92 & 0.63 & 0.14 & & 0.578 & 0.923 \\
\hline & $\mathrm{C} / \mathrm{K} 2: 2$ & 6.32 & 5.68 & 2.48 & 0.62 & 0.69 & 0.19 & & 21.325 & 0.882 \\
\hline & $\mathrm{C} / \mathrm{K} 2: 1: 1$ & 8.13 & 2.80 & 2.80 & 1.36 & 1.01 & 0.14 & & 19.219 & 0.869 \\
\hline Festuca & Obs. & 3.19 & 2.00 & 7.85 & 0.75 & 0.42 & 0.29 & 0.762 & & \\
\hline F. arundinacea & K/A 2:1:1 & 2.91 & 3.04 & 7.69 & 0.26 & 0.46 & 0.26 & & 1.424 & 0.970 \\
\hline PPXG & $\mathrm{C} / \mathrm{K} 2: 2$ & 1.45 & 4.28 & 6.94 & 0.15 & 0.50 & 0.42 & & 30.855 & 0.952 \\
\hline
\end{tabular}

*Obs. = observed numbers of each meiotic figure, averages per cell.

Sources of original data can be found in Kimber \& Alonso (1981), except example 4 from Kimber \& Yen (1988).

appreciably for entries 2 and 4 . In the case of the $T$. boeoticum $\times$ T. tauschii amphiploid, the higher value of $x$ estimated here suggests that pairing is more confined to within genomes than the earlier analysis indicated. For the T. triunciale $\times$ autotetraploid $T$. umbellulatum hybrid the converse is true, a smaller $x$ indicating some pairing between the $\mathrm{C}$ and $\mathrm{U}$ genomes necessary to accommodate the open quadrivalents observed.

The remaining hybrids are less clear-cut. In the fifth example, a hybrid between hexaploid $T$. crassum and diploid T. umbellulatum, the two approaches provide a similar result with the 2:1:1 model as the best fit, consistent with the pairing being predominantly between the two D genomes of $T$. crassum. For the sixth example, a $T$. juvenale $\times T$. longissimum hybrid, the two fits appear equally good but from the present analysis it seems three of the genomes are somewhat more similar to each other than the fourth, rather than all four being equally dissimilar. The analysis does not, of course, suggest which three might be, nor does the pedigree of the hybrid provide any guidance.

For the bread wheat $\times$ rye hybrid (example 7 ), both approaches give a $3: 1$ fit, consistent with the three Triticum genomes, $\mathrm{ABD}$, pairing to the exclusion of the $\mathrm{R}$ of Secale. The present analysis, however, suggests that this is not as exclusive as has been thought. This arises from the new 3:1 model's sensitivity to the number of quadrivalents leading to optimization on a lower value of $x$ to account for them. By contrast the 3:1 model of Kimber \& Alonso (1981) shows quadrivalents as being dependent on the value of $c$ alone and not, therefore, being taken into account when $x$ is estimated.

These last two examples also serve to emphasize one important aspect of all these models. $x$ is a measure of relative, not absolute, affinity. The high value of $x$ in example six only indicates that three of the genomes are similar when compared to the fourth, not that they 
should necessarily be considered the same genome. Similarly, the A and B genome only appear closely related in example seven when compared to the more distant rye; when compared to two sets of the D genome in example two they seem unrelated.

The case of the T. timopheevii $\times$ T. turgidum hybrid is more complex. Both analytical approaches favour a 2:1:1 solution, and the Kimber/Alonso analysis gives a fairly good fit. The present analysis gives a poorer fit and suggests that neither a 2:1:1 nor a $2: 2$ model is a good representation of the combined meiotic behaviour of the AABG genomes. A further analysis, reported on in a following paper (Chapman \& Kimber, $1992 \mathrm{~b})$ suggests that the meiotic behaviour of this hybrid is generally intermediate between the 2:1:1 and $2: 2$ models.

The final example is a hybrid which, under the present analysis, cannot fit the 2:1:1 model because the value of $c$ is too high. This illustrates a weakness of the Kimber/Alonso 2:1:1 model because, with an $x$ of 0.970 , pairing should be virtually confined to two genomes only and $c$ should be close to 0.5 . Instead, it appears that the four genomes are pairing two by two, although the fit is certainly poor, with the calculated figure numbers being too high for rod bivalents and ring quadrivalents, but low for univalents, ring bivalents and trivalents. Nevertheless it tends to lend support to the older view of Chandrasekharan \& Thomas (1971) that the genomes of Festuca arundinacea should be designated $P P G_{1} G_{1} G_{2} G_{2}$ rather than PPGGXX.

Comparisons may also be made with the more complex analysis for tetraploid hybrids of Crane \& Sleper (1989). This is 'overparametized', using more variables than there are degrees of freedom to account for them. Hence, it must seek permissible ranges for these variables rather than single best-fit solutions. Moreover, there is an immense computational burden requiring the use of one mini- or twelve microcomputers. By contrast the present analysis uses one microcomputer and takes between 2 and $3 \mathrm{~s}$ to run.

A comparison between the two methods can be made by estimating the values of $s_{1} \ldots s_{6}$ of Crane \& Sleper (1989), which measure the ratio of pairing amongst the four genomes, from the $x$ and $y$ values calculated here. Seven examples are presented in Table 4, being hybrids which are also to be found in Table 3.

Reasonable matches are found in the first five entries, where the analyses in Table 3 gave good fits

Table 4 A comparison between the present analysis and that of Crane \& Sleper (1989)

\begin{tabular}{|c|c|c|c|c|c|c|c|}
\hline Hybrid and genomes & Analysis & $s_{1}$ & $s_{2}$ & $s_{3}$ & $s_{4}$ & $s_{5}$ & $s_{6}$ \\
\hline Autotetraploid & $\mathrm{C} / \mathrm{S}$ high & 0.167 & 0.167 & 0.167 & 0.167 & 0.167 & 0.167 \\
\hline Hordeum bulbosum & C/S low & 0.167 & 0.167 & 0.167 & 0.167 & 0.167 & 0.167 \\
\hline BBBB & $\mathrm{C} / \mathrm{K}$ & 0.167 & 0.167 & 0.167 & 0.167 & 0.167 & 0.167 \\
\hline T. boeoticum $\times$ & $\mathrm{C} / \mathrm{S}$ high & 0.494 & 0.036 & 0.014 & 0.018 & 0.019 & 0.474 \\
\hline T. tauschii amph. & C/S low & 0.473 & 0.017 & 0.000 & 0.000 & 0.005 & 0.452 \\
\hline AADD & $\mathrm{C} / \mathrm{K}$ & 0.473 & 0.014 & 0.014 & 0.014 & 0.014 & 0.473 \\
\hline T. aestivum $\times$ & $\mathrm{C} / \mathrm{S}$ high & 1.000 & 0.000 & 0.000 & 0.000 & 0.000 & 0.000 \\
\hline T. tauschii & C/S low & 1.000 & 0.000 & 0.000 & 0.000 & 0.000 & 0.000 \\
\hline $\mathrm{ABDD}$ & $\mathrm{C} / \mathrm{K}$ & 1.000 & 0.000 & 0.000 & 0.000 & 0.000 & 0.000 \\
\hline T. crassum $\times$ & C/S high & 0.701 & 0.220 & 0.011 & 0.095 & 0.025 & 0.082 \\
\hline T. umbellulatum & C/S low & 0.679 & 0.103 & 0.000 & 0.000 & 0.010 & 0.074 \\
\hline DDMU & $\mathrm{C} / \mathrm{K}$ & 0.688 & 0.062 & 0.062 & 0.062 & 0.062 & 0.062 \\
\hline T. juvenale $\times$ & C/S high & 0.297 & 0.286 & 0.113 & 0.272 & 0.074 & 0.066 \\
\hline T. longissimum & $\mathrm{C} / \mathrm{C}$ low & 0.271 & 0.260 & 0.057 & 0.226 & 0.043 & 0.033 \\
\hline DMUS $^{l}$ & $\mathrm{C} / \mathrm{K}$ & 0.274 & 0.274 & 0.059 & 0.274 & 0.059 & 0.059 \\
\hline T. aestivum $\times$ & $\mathrm{C} / \mathrm{S}$ high & 0.621 & 0.396 & 0.095 & 0.149 & 0.116 & 0.000 \\
\hline S. cereale & $\mathrm{C} / \mathrm{S}$ low & 0.506 & 0.160 & 0.000 & 0.023 & 0.000 & 0.000 \\
\hline ABDR & $\mathrm{C} / \mathrm{K}$ & 0.297 & 0.297 & 0.038 & 0.297 & 0.038 & 0.038 \\
\hline T. timopheevii $\times$ & C/S high & 0.656 & 0.149 & 0.001 & 0.069 & 0.000 & 0.212 \\
\hline T. turgidum & $\mathrm{C} / \mathrm{S}$ low & 0.646 & 0.071 & 0.000 & 0.000 & 0.000 & 0.204 \\
\hline $\mathrm{AABG}$ & $\mathrm{C} / \mathrm{K}$ & 0.570 & 0.086 & 0.086 & 0.086 & 0.086 & 0.086 \\
\hline
\end{tabular}

$s_{1} \ldots s_{6}=$ estimates of metaphase I association due to each combination of genomes.

$\mathrm{C} / \mathrm{S}$ high $=$ Crane \& Sleper's highest estimate of $s_{1} \ldots s_{6}$.

$\mathrm{C} / \mathrm{S}$ low $=$ Crane \& Sleper's lowest estimate of $s_{1} \ldots s_{6}$.

$\mathrm{C} / \mathrm{K}=$ present estimate of $s_{1} \ldots s_{6}$ from $x$ and $y$ of the best fitting model.

Data sources as in Table 3. 
between observed and calculated figure numbers. In the case of wheat by rye hybrid (example 6) $s_{1}$ appears underestimated and $s_{4}$ overestimated. Kimber \& Alonso (1981) suggest that among the wheat genomes A may pair preferentially with $D$; i.e. this hybrid is intermediate between the $2: 1: 1$ and $3: 1$ models. This would account for the discrepancies noted above. The seventh example, $T$. timopheevii $\times T$. turgidum, has also been noted as an intermediate case, this time between the $2: 2$ and $2: 1: 1$ models, hence the underestimate of $s_{6}$.

It is true that the present analyses present only three types of model to accommodate a wide range of possible genomic relationships. These simple models, however, explain much of the meiotic behaviour observed. We believe, as in the case of the triploids, that the complexity of Crane \& Sleper's approach is difficult to justify when the simplifications it still contains (that the chromosomes in each homeologous group are similarly differentiated and that translocations are absent) and the inherent quality of meiotic data are considered.

In conclusion, the amended models for meiosis in hybrids, developed here, better reflect the theoretical properties such models should have than their antecedents in Kimber \& Alonso (1981). Where hybrids are known a priori to be good examples of particular models, the present analyses generally give a better fit between observed and calculated figure numbers. In other examples the fit may be better or worse, and may also lead to differing conclusions about the general meiotic behaviour of the hybrid in question.

\section{A note on programs}

All the programs for the analysis of meiosis in species hybrids, described in this series of papers, have been written in Turbo Pascal to run on the Apple Macintosh. Copies of the source code and the compiled programs are available from the authors upon reciept of an 800 $\mathrm{K}$ or $1.4 \mathrm{MB}$ initialized disk. Use of the source code requires Turbo Pascal.

\section{Acknowledgements}

CGDC was supported by a post-doctoral fellowship from the University of Missouri. Contribution from the
Missouri Agricultural Experiment Station. Journal Series Number: 11,258.

\section{References}

ALONSO, L. C. AND KIMBER, G. 1981. The analysis of meiosis in hybrids. II. Triploid hybrids Can. J. Genet. Cytol., 23, 221-234.

CHANDRASEKHARAN, P. AND THOMAS, H. 1971. Studies in Festuca. 6. Chromosome relationships between Bovinae and Scariosae. Z. Pflanzenzüchtg., 66, 76-86.

CHAPMAN, C. G. D. AND KIMBER, G. 1992a. Developments in the meiotic analysis of hybrids. I. Review of theory and optimization in triploids. Heredity, 68, 97-103.

CHAPMAN, C. G. D. AND KIMBER, G. 1992b. Developments in the meiotic analysis of hybrids. V. Second order models for tetraploids and pentaploids. Heredity, 68, (in press).

CRANE, C. F. AND SLEPER, D. A. 1989. A model of meiotic chromosome association in tetraploids. Genome, 32, 691-707.

DRISCOLL, C. J., BIELIG, L. M. AND DARVEY, N. L. 1979. An analysis of frequencies of chromosome configurations in wheat and wheat hybrids. Genetics, 91, 755-767.

ESPINASSE, A. AND KIMBER, G. 1981. The analysis of meiosis in hybrids. IV. Pentaploid hybrids. Can. J. Genet. Cytol., 23, 627-638.

JACKSON, R. C. AND CASEY, J. 1982. Cytogenetic analyses of autopolyploids: models and methods for triploids to octoploids. Am. J. Bot., 69, 485-501.

KIMBER, G. AND ALONSO, L. C. 1981. The analysis of meiosis in hybrids. II. Tetraploid hybrids. Can. J. Genet. Cytol., 23, 235-254.

KIMBER, G., ALONSO, L. C. AND SAlleE, P. J. 1981. The analysis of meiosis in hybrids. I. Aneuploid hybrids. Can. J. Genet. Cytol., 23, 191-201.

KIMBER, G., PIGNONE, D. AND SALLEE, P. J. 1983. The relationships of the $\mathrm{M}$ and $\mathrm{M}^{\mu}$ genomes of Triticum. Can. J. Genet. Cytol., 25, 509-512.

KIMBER, G. AND TSUNEWAKI, K. 1988. Genome symbols and plasma types in the wheat group. Proc. 7th Intern. Wheat Genet. Symp., Cambridge, U.K., 1209-1211.

KIMBER, G. AND YEN, Y. 1988. Hybrids involving wheat relatives and autotetraploid Triticum umbellulatum. Genome, 32, $1-5$.

LILIENFELD, F. A. 1951. H. Kihara: genome-analysis in Triticum and Aegilops. X. Concluding review. Cytologia, 16, 101-123. 